\title{
Prevalence and impact of chronic widespread pain in the Bangladeshi and White populations of Tower Hamlets, East London
}

\author{
Yasmin Choudhury • Stephen A. Bremner • Anwara Ali • Sandra Eldridge • \\ Chris J. Griffiths • Iqbal Hussain • Suzanne Parsons • Anisur Rahman • Martin Underwood
}

Received: 19 March 2013 / Accepted: 30 April 2013 / Published online: 30 May 2013

(C) The Author(s) 2013. This article is published with open access at Springerlink.com

\begin{abstract}
The prevalence and impact of chronic pain differ between ethnic groups. We report a study of the comparative prevalence and impact of chronic pain in Bangladeshi, British Bangladeshi and White British/Irish people. We posted a short questionnaire to a random sample of 4,480 patients registered with 16 general practices in the London Borough of Tower Hamlets and conducted a longer questionnaire with patients in the waiting areas at those practices. We distinguished between Bangladeshi participants who were born in the UK or had arrived in the UK at the age of 14 or under (British Bangladeshi) and those who arrived in UK at the age of over 14 (Bangladeshi). We obtained
\end{abstract}

Electronic supplementary material The online version of this article (doi:10.1007/s10067-013-2286-3) contains supplementary material, which is available to authorized users.

Y. Choudhury $\cdot$ S. A. Bremner $(\bowtie) \cdot$ S. Eldridge $\cdot$ C. J. Griffiths Barts and The London School of Medicine and Dentistry, Centre for Primary Care and Public Health, 58 Turner Street, London E1 2AB, UK

e-mail: s.a.bremner@qmul.ac.uk

A. Ali

The Spitalfields Practice, London, UK

I. Hussain

East London NHS Foundation Trust, London, UK

S. Parsons

Nowgen, Manchester Academic Health Science Centre, Central Manchester University Hospitals NHS Foundation Trust and The University of Manchester, Manchester, UK

A. Rahman

University College Hospital, London, UK

M. Underwood

Health Sciences Research Institute, University of Warwick,

Coventry, UK
$1,223 / 4,480(27 \%)$ responses to the short survey and $600 / 637$ (94 \%) to the long survey. From the former, the prevalence of chronic pain in the White, British Bangladeshi and Bangladeshi groups was 55, 54 and $72 \%$, respectively. The corresponding figures from the long survey were 49,45 and $70 \%$. Chronic widespread pain was commoner in the Bangladeshi (16\%) than in the White (10\%) or British Bangladeshi (9\%) groups. People with chronic pain experienced poorer quality of life (odds ratio for scoring best possible health vs. good health (or good vs. poor health) 5.6 (95\% confidence interval 3.4 to 9.8)), but we found no evidence of differences between ethnic groups in the impact of chronic pain on the quality of life. Chronic pain is commoner and, of greater severity, in Bangladeshis than in Whites. On most measures in this study, British Bangladeshis resembled the Whites more than the Bangladeshis.

Keywords Chronic pain · Comparative prevalence · Ethnicity $\cdot$ Quality of life

\section{Introduction}

Chronic pain has large direct and indirect health-care costs and a major impact on people's health. Prevalence estimates vary widely from 5.2 to $10.5 \%$, possibly because of variations in the study design and definitions of pain. Chronic widespread pain prevalence is reported to be between 1 and $15 \%$, and chronic regional pain, between 20 and $25 \%$ [1, 2].

Anecdotally, managing chronic pain in the people of South Asian origin living in the UK is challenging. Developing a strategy to manage this perceived problem requires the study of the prevalence and health impact of chronic pain in different ethnic groups. We were particularly 
interested in the management of chronic pain in the Borough of Tower Hamlets in East London, the third most deprived borough in England [3] with $31 \%$ of residents born outside the EU [4]. Most of the population are either White British/Irish (henceforth, 'White') or Bangladeshi. Nearly 66,000 people gave their ethnic group as Bangladeshi or British Bangladeshi in the 2001 census [3], and the majority come from Sylheti in the north-east Bangladesh and speak Sylheti, a separate dialect of Bengali. A study in Tower Hamlets found complex and multiple routes of care and referral systems for chronic pain patients, and a need for a better management of chronic pain [5] and a key part of this are defining whether the features and effects of chronic pain are similar or different in the Bangladeshi and White populations.

Few studies have measured pain across different ethnic groups in the UK, and these studies have not focused on Bangladeshis. One study in Greater Manchester found that there were substantially higher proportions of South Asian individuals (Indians, Pakistanis and Bangladeshis) reporting regional and widespread pain relative to the local European population [6]. A more recent study in the Manchester area [7] found that the odds of widespread pain were higher in South Asians than those in Europeans (odds ratio 3.7, $95 \%$ confidence interval (CI) 2.9 to 4.9), and in South Asians, widespread pain was associated with lower acculturation (adoption of the views and customs of the host community). However, this study looked at pain present for at least $24 \mathrm{~h}$ in the previous month rather than chronic pain (usually defined as pain being present for 3 months or more). Furthermore, not all South Asian groups were equally represented in the study. Here, we report a study to compare the prevalence and features of chronic pain in the White and Bangladeshi populations in Tower Hamlets.

\section{Method}

We recruited subjects for a postal (short) and a face-to-face (long) survey from 16 general practices within Tower Hamlets. Ethical approval for the study was granted by the East London and the City Research Ethics Committee (REC reference number 05/Q0605/185).

In the pilot work, the response rate to detailed postal questionnaires in Tower Hamlets was unacceptably low (12\%) [8]. To address this, we used a short postal/telephone questionnaire to obtain comparative data on the prevalence and distribution of pain, and a longer questionnaire was administered face to face in general practice waiting rooms to a different sample, which also addressed the health impact of chronic pain. We recognised at the design stage that response rates in the Tower Hamlets area would be too low to make robust estimates of the prevalence of pain in the population. Rather, we were interested in the comparative prevalence and health impact between different groups who share many of the characteristics associated with a poor response rate to surveys.

Since acculturation may affect the prevalence of widespread pain, and the level of acculturation increases with the amount of time South Asians have been in the UK, we distinguished in our analyses between Bangladeshis who had arrived in the UK before the age of 14 and those who had arrived after this age [7]. We assumed that those who migrated after the age of 14 would have had most of their education and formative experiences in Bangladesh and would thus have lower acculturation than those who migrated earlier. The distribution of age of entry to UK of our Bangladeshi and British Bangladeshi subjects was bimodal with peaks at birth/infancy and early adulthood with a trough around the age of 14-16, providing empirical support for this theoretically derived cut-point. The decision to divide our sample into three groups was made prior to any analyses. All analyses compared these three groups.

\section{Short (postal) survey}

Each participating practice selected a random sample of patients aged 18 years and over from the practice register. To detect a relative risk of chronic widespread pain of 2.0 between two equally sized groups with $80 \%$ power at the $5 \%$ significance level (assuming baseline prevalence of $10 \%$ ), we require data on 219 individuals in each group. To allow for clustering by practice, with an intra-cluster correlation coefficient of 0.05 , we assume 30 per group in each practice results in 537 individuals required in each group. We originally planned a two-group comparison (Whites and Bangladeshis), assuming that these two groups would comprise $60 \%$ of the total population, thus that we needed 1,790 individuals to respond to our questionnaires. Assuming practices would exclude $20 \%$ of those selected patients, and only $50 \%$ of those approached would respond; we required 4,475 individuals from 18 practices to be selected. To ensure sufficient responses from older Bangladeshi residents, who are expected to be less likely to respond to postal questionnaires because of poor literacy, within each practice, we selected two random samples: one of 175 patients aged 18-35 and one of 175 patients aged 36 and over. Practices could then exclude patients with pain due to an established patho-physiological diagnosis, who were terminally ill or whom the practice considered it inappropriate to approach for any other reason. Researchers posted a two-page questionnaire (see Web Table 1) and covering letter from each practice to everyone selected; for those with Asian surnames, a Bengali version of the letter and questionnaire was also included. 
After 2 weeks, the researchers telephoned non-responders, inviting them to complete the same questionnaire over the telephone. All calls and telephone administration of the questionnaire were made in either English or in Sylheti using preagreed phonetic translations.

Those from other ethnicities also received and responded to this questionnaire. We present a descriptive analysis of the data from these individuals, but they are not included in the detailed analyses focused on the White and Bangladeshi groups.

\section{Long (face-to-face) survey}

This questionnaire, carried out in the waiting rooms of general practices, was 12 pages long (see Web Table 2 for items collected). All items were translated into Bengali.

Data on approximately 150 individuals would allow us to include ten covariates as explanatory variables in a regression model with quality of life as the dependent variable. We inflated our sample size to account for clustering by practice. Assuming 40 patients would be recruited from each participating practice and an intra-cluster correlation coefficient of 0.05 , we inflated our sample size by a factor of 2.95 . Thus, we needed to recruit a minimum of 442 (i.e. $150 \times 2.95$ ) participants. However, to allow for variable recruitment rates by practice and other unforeseen circumstances, we planned to recruit 40 participants from each of 16 practices.

We used quota sampling to ensure roughly equal representation of males and females, the numbers of people aged under and over 35 which are proportional to the numbers on the practice list and the number of White and Bangladeshi subjects which is proportional to the 2001 Census breakdown for the Local Authority Ward in which the practice was located. White and Bangladeshi individuals were approached in the waiting areas of the 16 general practices by either the male or female bilingual (Sylheti and English) researcher involved in the study and were asked whether they were willing to take part. The researchers completed the questionnaires for those unable to do so.

\section{Analysis}

\section{Prevalence and site of pain}

We determined the presence of chronic pain from a question in both questionnaires which asked whether participants had had pain for three months or longer [9]. To determine the presence of chronic widespread pain, defined as 'pain in two contralateral quadrants of the body and in the axial skeleton that has been present for at least three months [10]', we used pain manikin data indicating areas where patients had had pain present for at least half of the days in the past year [11]. Because of these differences in time frame, there were participants who had chronic pain but did not complete the manikin.
We used the short survey data to compare the prevalence of chronic pain in the three ethnic groups, using logistic regression fitted by generalised estimating equations to take account of the clustering by practice. We adjusted for the following potentially confounding factors: age, sex, age on leaving full-time education (categorised as follows: by 12 years of age/no formal education, between 12 and 16 years of age (reference group) and 17 to 20 years of age or beyond/still in full-time education), employment status and ethnic origin.

\section{Health impact of chronic pain}

For the health impact analyses, we used the long survey data. We measured health impact using the EQ-5D score [12], divided into three groups for analysis: best possible health (patients scoring 1, reference group), medium health (patients scoring from 0.50 to less than 1) and poor health (patients scoring less than 0.50 ). The bimodal, left-skewed distribution of EQ-5D means modelling as a continuous variable [13, 14] is complicated, and we preferred a method more easily accessible to readers. To assess the health impact of chronic pain, we fitted an ordered logistic regression model to the grouped EQ-5D scores, with standard errors robust to clustering and chronic pain as a predictor. We tested the proportional odds assumption (that the odds ratio comparing the group in poorest health to the middle group is of the same magnitude as the odds ratio comparing the middle group to the best possible health group) using the Brant test [15]. The model was adjusted for the same confounders as in the pain prevalence model. To assess whether the health impact of pain was different between different ethnic groups, we fitted a statistical interaction between ethnic group and chronic pain. All analyses were carried out in Stata version 10.1 [16].

\section{Results}

Response rate and demographic characteristics of responders

We collected data from mid-2007 to early 2008 . We obtained 1,223/4,480 (27\%) responses to the short survey and 600 responses (94\% of those approached) to the long survey (Table 1). Approximately $50 \%$ of respondents in each survey were White. In the short survey, $12 \%$ were British Bangladeshi; $16 \%$, Bangladeshi; and $25 \%$, of other ethnicities (Table 1). In the long survey, proportions in each ethnic group were in accordance with the quota sampling. White respondents in the long survey were, on average, 10 years younger than those in the short survey. The mean age of respondents was similar in each of the Bangladeshi 
Table 1 Sample characteristics and prevalence of chronic pain, by ethnic origin in the short telephone/postal and long waiting room surveys

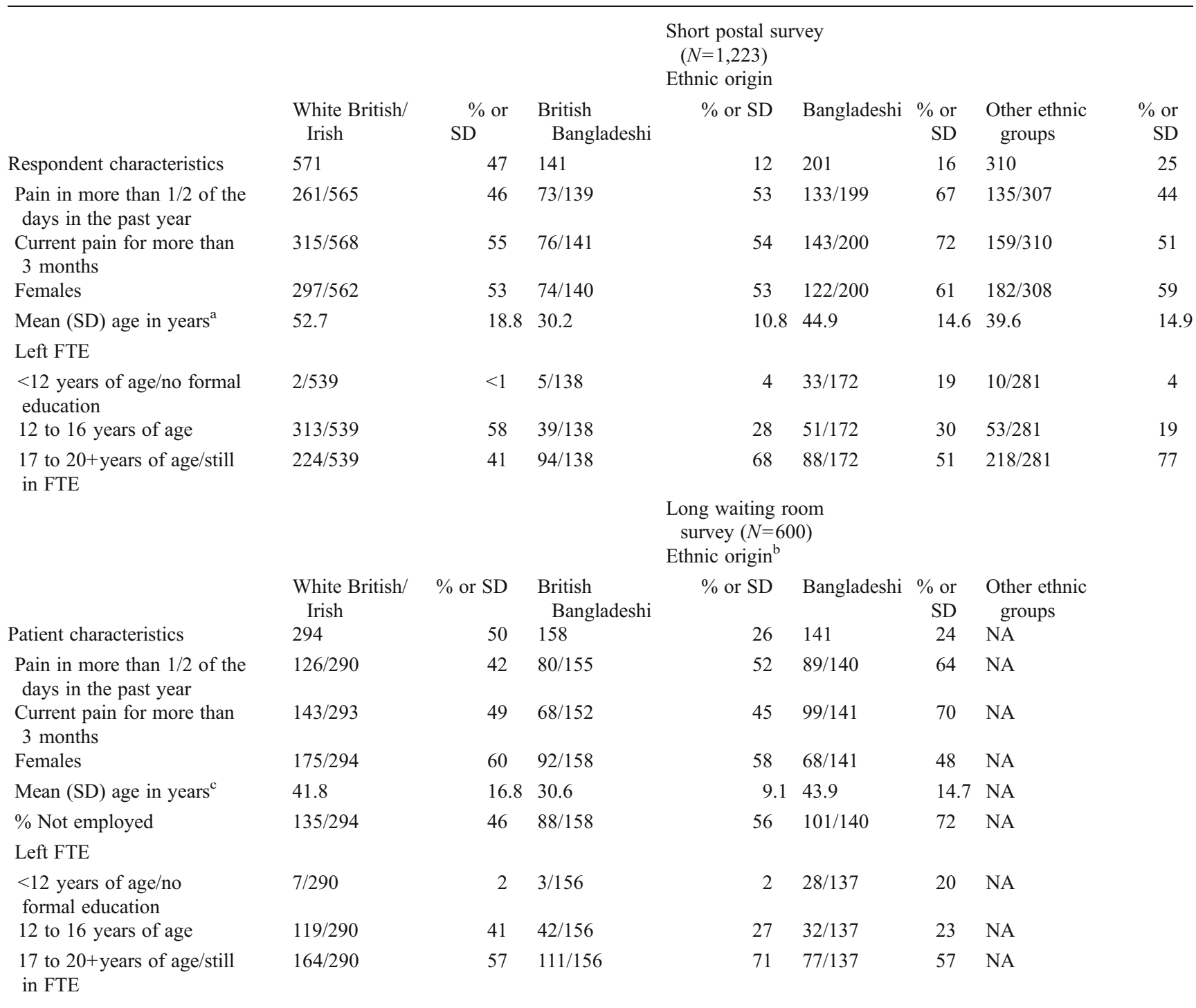

$S D$ standard deviation, FTE full-time education, $N A$ not applicable

${ }^{\text {a }}$ Age is missing on 3 respondents

${ }^{\mathrm{b}}$ Missing on 7 individuals

${ }^{\mathrm{c}}$ Age is missing on 17 patients

groups between the surveys. The majority of White longsurvey respondents were in employment, whilst a minority of the Bangladeshi respondents were unemployed. Approximately one fifth of Bangladeshis did not stay in fulltime education beyond the age of 12 , a much larger proportion than in the White or British Bangladeshi samples.

Prevalence and site of chronic pain in different ethnic groups

The prevalence of chronic pain (Table 2) was 56/49\% (short/long survey) in the White population and 54/45\% amongst British Bangladeshis, but higher in Bangladeshis (72/70 \%). Similarly, both surveys recorded higher proportions of chronic widespread pain in the Bangladeshis $(16 / 18 \%)$ than in the White (10/6 \%) or British Bangladeshi $(9 / 9 \%)$ groups. This marked similarity in profile between the two surveys gives us some degree of confidence in extrapolating from data collected in the long survey to the population as a whole.

Pain distribution data were available on $49 \%$ of the short survey and $47 \%$ of long survey participants. There is a striking agreement between the results of the short and long surveys within individual groups (comparing manikin 1 
Table 2 Prevalence (prop.) of chronic pain (short and long surveys) and predictors of chronic pain from (short survey)

\begin{tabular}{|c|c|c|c|c|c|c|c|}
\hline \multirow[b]{2}{*}{ Ethnic origin } & \multirow[b]{2}{*}{ Survey } & \multirow[b]{2}{*}{ Prop. } & \multicolumn{2}{|l|}{ Chronic pain } & \multirow[b]{2}{*}{ Prop. } & \multicolumn{2}{|c|}{ Chronic widespread pain ${ }^{\mathrm{b}}$} \\
\hline & & & $95 \%$ CI for prop. & $n$ & & $95 \%$ CI for prop. & $n$ \\
\hline \multirow[t]{2}{*}{ White British/Irish } & Short & 0.56 & 0.51 to 0.60 & 571 & 0.10 & 0.02 to 0.18 & 58 \\
\hline & Long & 0.49 & 0.43 to 0.55 & 294 & 0.06 & $0.00^{\mathrm{a}}$ to 0.18 & 19 \\
\hline \multirow[t]{2}{*}{ British Bangladeshi } & Short & 0.54 & 0.46 to 0.62 & 141 & 0.09 & $0.00^{\mathrm{a}}$ to 0.25 & 13 \\
\hline & Long & 0.45 & 0.37 to 0.53 & 158 & 0.09 & $0.00^{\mathrm{a}}$ to 0.24 & 13 \\
\hline \multirow[t]{2}{*}{ Bangladeshi } & Short & 0.72 & 0.65 to 0.78 & 201 & 0.16 & 0.03 to 0.28 & 31 \\
\hline & Long & 0.70 & 0.62 to 0.77 & 141 & 0.18 & 0.03 to 0.33 & 25 \\
\hline \multirow[t]{3}{*}{ other ethnic groups } & Short & 0.51 & 0.46 to 0.57 & 310 & 0.09 & $0.00^{\mathrm{a}}$ to 0.20 & 29 \\
\hline & Survey & \multicolumn{3}{|c|}{$\begin{array}{l}\text { Chronic pain vs. no pain } \\
\text { ( } 835 \text { respondents) }\end{array}$} & \multicolumn{3}{|c|}{$\begin{array}{l}\text { Chronic widespread pain }{ }^{\mathrm{b}} \text { vs. chronic pain } \\
\text { (484 respondents) }\end{array}$} \\
\hline & & OR & $95 \%$ CI for OR & $P$ value & OR & $95 \% \mathrm{CI}$ for $\mathrm{OR}$ & $P$ value \\
\hline $\begin{array}{l}\text { British Bangladeshi vs. White } \\
\text { British/Irish }\end{array}$ & Short & 1.84 & 1.15 to 2.94 & 0.01 & 1.80 & 0.65 to 5.04 & 0.26 \\
\hline Bangladeshi vs. White British/Irish & Short & 2.68 & 1.53 to 4.69 & 0.001 & 1.47 & 0.58 to 3.77 & 0.42 \\
\hline \multicolumn{8}{|l|}{ Age at which left FTE } \\
\hline $\begin{array}{l}\text { Left FTE at }<12 \text { years/no formal } \\
\text { education vs. left FTE } 12-16 \text { years }\end{array}$ & Short & 0.80 & 0.43 to 1.46 & 0.46 & 1.16 & 0.35 to 3.82 & 0.80 \\
\hline $\begin{array}{l}\text { Left FTE } 17-20+\text { years/still in FTE } \\
\text { vs. left FTE } 12-16 \text { years }\end{array}$ & Short & 0.55 & 0.39 to 0.78 & 0.001 & 0.45 & 0.24 to 0.86 & 0.02 \\
\hline Age (years) & Short & 1.02 & 1.01 to 1.04 & 0.003 & 1.01 & 0.99 to 1.04 & 0.16 \\
\hline Sex (male vs. female) & Short & 0.99 & 0.77 to 1.28 & 0.95 & 1.04 & 0.65 to 1.68 & 0.86 \\
\hline
\end{tabular}

$95 \%$ CI $95 \%$ confidence interval, $n$ number of respondents, OR odds ratio, FTE full-time education

${ }^{\mathrm{a}}$ Lower confidence limit truncated at zero

${ }^{\mathrm{b}}$ The American College of Rheumatology definition

with 2, 3 with 4 and 5 with 6) (Fig. 1). From the short survey, the commonest site of pain was the lower back in all three groups. Bangladeshis have more sites where the prevalence of chronic pain exceeds $10 \%$ than either the White or British Bangladeshi groups (manikins 5 and 6). Detailed pain prevalence estimates are presented in Web Table 3.

Factors associated with chronic pain

Confirming univariate results, a multiple logistic regression model (Table 2) fitted to the data from the short survey showed that chronic pain was positively associated with Bangladeshi ethnic origin (vs. White), odds ratio $(\mathrm{OR})=$ 2.68 (95\% CI 1.53 to 4.69); British Bangladeshi origin (vs. White), $\mathrm{OR}=1.84$ (95\% CI 1.15 to 2.94 ); and age, $\mathrm{OR}=1.02$ (95\% CI 1.01 to 1.04$)$. Those who left full-time education (FTE) at the age of 17 or older or who were still enrolled had a lower odds of chronic pain than respondents who had left FTE by the age of 16 (OR $0.55,95 \%$ CI 0.39 to 0.78 ). There was no evidence of an association between reporting chronic pain and being male vs. female, $\mathrm{OR}=0.99$, $95 \% \mathrm{CI}(0.77$ to 1.28$)$. A further multiple logistic regression model (Table 2) showed that amongst those with chronic pain, patients who had remained in FTE beyond the age of 16 had a lower odds of widespread pain.

The health impacts of chronic pain

The median EQ-5D index score was lower in the Bangladeshi group (0.69) than in the other groups, 0.80 in each (Table 3). The mean 12-item General Health Questionnaire (GHQ12) scores were similar between the three ethnic origin groups (Table 3). A similar pattern was observed for pain intensity and disability scores (Table 3).

The proportional odds were 5.8 times greater in patients with chronic pain compared to patients reporting no pain, $95 \%$ CI (3.4 to 9.8) (Table 4). Compared to the Whites, neither the British Bangladeshi group (proportional odds ratio $(\mathrm{POR})=1.01,95 \%$ CI 0.55 to 1.80 ) nor the Bangladeshi group ( $\mathrm{POR}=1.07,95 \% \mathrm{CI} 0.49$ to 2.32 ) had significantly different odds of being in a worse category of the EQ-5D index. We found no evidence that the effect of chronic pain on health-related quality of life was modified by ethnic group (composite $P$ value for interaction terms of 0.25 ), although our study was not powered to detect such an effect. 


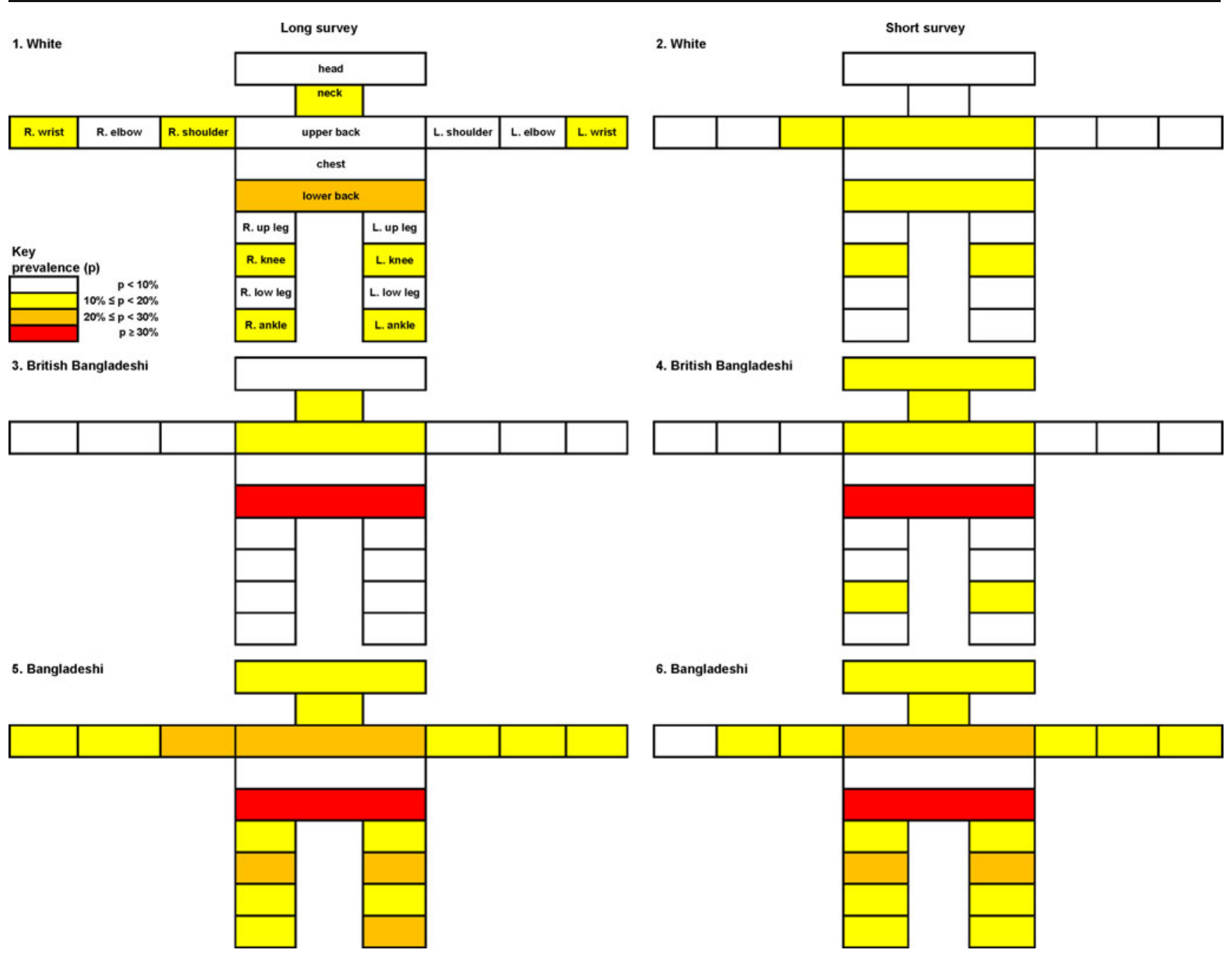

Fig. 1 Pain manikins (viewed from front) depicting the prevalence of pain in at least half of the days in the past year

\section{Discussion}

To our knowledge, this is the first study to make a direct comparison of the prevalence and characteristics of chronic musculoskeletal pain in White and Bangladeshi people living in the same geographical area and attending the same general practices. Unlike Palmer et al. [7], we found a high prevalence of pain in the Bangladeshis from Sylheti-though it should be noted that they were studying widespread pain rather than chronic pain and did find a high prevalence of this in non-Sylheti Bangladeshis. Like them, however, our results are consistent with the view that Bangladeshis who have lower levels of acculturation have more pain. We did not use a formal measure of acculturation but identified a group who arrived in the UK after their 14th birthday, were particularly likely to have left full-time education before the age of 12 and had a higher prevalence of both chronic pain and chronic widespread pain than those of the other groups shown in this study; $70 \%$ of this group reported chronic pain. The health-related quality-of-life impact of chronic pain was severe with sufferers having sixfold increased odds of poor quality of life on EQ-5D. Interestingly, patients in the three groups scored similarly in the GHQ12 questionnaire. The Whites had the lowest median pain intensity and disability scores; British Bangladeshis had slightly higher scores, and the Bangladeshis had the highest scores. The Bangladeshi group had lower EQ-5D scores than the other groups (see Table 3), as might be expected from the higher prevalence of chronic pain within this group, although the number of subjects studied was too low to detect a statistically significant difference between ethnic groups.

An important feature of this study was our use of two separate sampling methods for obtaining data. The methods are complementary, having different advantages and disadvantages. Responses to the short postal survey (47\% white, $28 \%$ Bangladeshi and $25 \%$ other ethnic groups) reflected the distribution of the adult Tower Hamlets population in 2006 (50\% White, $25 \%$ Bangladeshi and $25 \%$ from other ethnic groups) [17]. 
Table 4 Predictors of the EuroQol Index category

\begin{tabular}{llll}
\hline & \multicolumn{2}{l}{ (528 patients) } & \\
\cline { 2 - 4 } & POR & $95 \%$ CI & $P$ value \\
\hline Chronic pain vs. no pain & 5.75 & 3.38 to 9.77 & $<0.001$ \\
British Bangladeshi vs. White British & 1.01 & 0.55 to 1.80 & 0.72 \\
Bangladeshi vs. white British & 1.07 & 0.49 to 2.32 & 0.78 \\
Chronic pain $\times$ British Bangladeshi & 1.54 & 0.66 to 3.61 & 0.32 \\
Chronic pain $\times$ Bangladeshi & 2.13 & 0.83 to 5.49 & 0.12 \\
Not employed vs. employed & 2.06 & 1.38 to 3.08 & $<0.001$ \\
Left FTE at <12 years/no formal & 2.23 & 0.94 to 5.31 & 0.07 \\
$\quad$ education vs. left FTE 12-16 years & & & \\
Left FTE 17 to 20+years/still in FTE & 0.76 & 0.50 to 1.17 & 0.21 \\
$\quad$ vs. left FTE 12-16 years & 1.02 & 1.01 to 1.04 & 0.001 \\
Age (years) & 1.65 & 1.13 to 2.43 & 0.01 \\
Sex (male vs. female) & & & \\
\hline
\end{tabular}

The reference category indicates perfect health (index score 1); category 1 indicates good health $(0.5 \leq$ index score of $<1)$, and category 2 , poor health $(<0.5)$

POR proportional odds ratio, $95 \%$ CI $95 \%$ confidence interval

Although both survey methods have disadvantages, our similar results, using the two different methods, provides an element of triangulation which strengthens the validity of the results. We suggest that a similar approach might be taken in other communities where postal questionnaire studies are difficult.

The study had some limitations. It is possible that pain reporting was different in those completing the survey alone compared to those completing it with one of the researchers over the phone. Further, reporting over the phone is more prone to error in marking the pain manikins correctly as the patient cannot point out exactly where they are feeling the pain. As anticipated, we obtained a lower response rate in this deprived inner London borough than one might expect in some other localities. The study was, however, designed to show differences between those who responded to the survey from different ethnic groups rather than an absolute prevalence of pain. Thus, the low response rate does not materially affect the interpretation of our findings unless there is differential response bias. We found no evidence of this. The survey instruments used have not been validated in the Bangladeshi population. Standard scores for the EQ$5 \mathrm{D}$ items were calculated by applying weights which were estimated from a regression model on 2,997 British individuals surveyed in 1993 [20]. While this is likely to be broadly representative of the British population at that time, different weights might be appropriate for the Bangladeshi population in which very few of whom would have featured in the 1993 survey; this ethnic group comprised less than $0.5 \%$ of the 1991 Census population [21]. This may limit the applicability of the instrument to this population. Similar 
criticism could be levelled at the GHQ12 questionnaire and the chronic pain grade.

Our data indicate that chronic pain is both more common and more severe in Bangladeshis in Tower Hamlets than in either British Bangladeshis or Whites. There is a suggestion that this is also associated with reduced quality of life in that group. It is clear that, regardless of ethnicity, those with chronic pain have lower quality of life as measured by EQ-5D. Future work will need to explore the reasons for these differences. There is large unmet health need in both the White and Bangladeshi populations in Tower Hamlets that may need to be addressed by developing services tailored to the needs of different groups.

Acknowledgments We thank all the practices who took part in the study and all those involved with the study itself. This work was supported by the Arthritis Research Campaign UK grant number 17239.

Ethical approval The Tower Hamlets Pain Study (TOPAS) was approved by the East London and the City Research Ethics Committee.

\section{Disclosures None.}

Open Access This article is distributed under the terms of the Creative Commons Attribution License which permits any use, distribution, and reproduction in any medium, provided the original author(s) and the source are credited.

\section{References}

1. Ospina M, Harstall C (2002) Prevalence of chronic pain: an overview. Report: HTA 29. Alberta Heritage Foundation for Medical Research, Edmonton, Alberta, Canada

2. McBeth J, Jones K (2007) Epidemiology of chronic musculoskeletal pain. Best Practice and Research Clin Rheumatol 21:403-425

3. Office for National Statistics (2001) Census: neighbourhood ethnic minority statistics. Stationary Office, London

4. Tower Hamlets (2001) Ethnicity. http://www.towerhamlets.gov.uk/ lgst/901-950/916_borough_statistics/ethnicity.aspx. Accessed 2 Mar 2013

5. Carnes D, Parsons S, Ashby D et al (2007) Chronic musculoskeletal pain rarely presents in a single body site: results from a UK population study. Rheumatology 46:1168-1170

6. Allison T, Symmons D, Brammah T et al (2002) Musculoskeletal pain is more generalised among people from ethnic minorities than among white people in Greater Manchester. Ann Rheum Dis 61:151-156

7. Palmer B, Macfarlane G, Afzal C et al (2007) Acculturation and the prevalence of pain amongst South Asian minority ethnic groups in the UK. Rheumatology 46:1009-1014

8. Parsons S, Breen A, Foster N et al (2007) Prevalence and comparative troublesomeness by age of musculoskeletal pain in different body locations. Fam Pract 24:308-316

9. Choudhury Y, Hussain I, Parsons S et al (2012) Methodological challenges and approaches to improving response rates in population surveys in areas of extreme deprivation: comparison of two pilot studies and a nested randomised controlled trial of personal correspondence. Prim Health Care Res Dev. doi:10.1017/ S1463423611000466

10. International Association for the Study of Pain (1986) International Association for the Study of Pain. Classification of chronic pain. Pain Suppl 3:S1-S226

11. Wolfe F, Smythe H, Yunus M et al (1990) The American College of Rheumatology criteria for the classification of fibromyalgia: report of the Multicenter Criteria Committee. Arthritis Rheum 33:160-172

12. EuroQol Group (1990) EQ-5D health questionnaire. English version for the UK

13. Carnes D, Gallagher J, Herne S et al (2008) Mapping care pathways and estimating the number and cost of musculoskeletal chronic pain patients to inform the development and implementation of a new service. Prim Health Care Res Dev 9:241-244

14. Clarke P, Gray A, Holman R (2002) Estimating utility values for health states of type-2 diabetic patients using the EQ-5D (UKPDS 62). Med Decis Making 22:340-349

15. Bagust A, Beale S (2005) Modelling EuroQol health-related utility for diabetic complications from CODE-2 data. Health Econ 14:217-230

16. Brant R (1990) Assessing proportionality in the proportional odds model for ordinal logistic regression. Biometrics 46:1171-1178

17. A guide to comparing 1991 and 2001 Census ethnic groups. Office of National Statistics. GuideV9_tcm77_169094_1.pdf p42 Table 17. http://www.ons.gov.uk/. Accessed 21 May 2012

18. Goldberg D, Williams P (1991) A user's guide to the General Health Questionnaire. NFER-Nelson, Windsor

19. Smith BH, Penny KI, Purves AM et al (1997) The chronic pain grade questionnaire: validity and reliability in postal research. Pain $71: 141-147$

20. Dolan P, Gudex C, Kind P et al (1995) A social tariff for EuroQol: results from a UK General Population Survey. http:// www.york.ac.uk/inst/che/pdf/DP138.pdf. Accessed 14 Feb 2013

21. Neighbourhood Statistics (2008) Tower Hamlets (2001) Census area statistics for Tower Hamlets. (June 24). http:// www.neighbourhood.statistics.gov.uk

This paper has neither been offered nor is under consideration for publication elsewhere, and neither the work nor the abstract of it has been previously published. 